Natural Hazards and Earth System Sciences, 5, 293-299, 2005

SRef-ID: 1684-9981/nhess/2005-5-293

European Geosciences Union

(C) 2005 Author(s). This work is licensed

under a Creative Commons License.

\title{
Terrain modelling by kinematical GPS survey
}

\author{
G. Nico ${ }^{1}$, P. Rutigliano ${ }^{2}$, C. Benedetto ${ }^{3}$, and F. Vespe $^{4}$ \\ ${ }^{1}$ Istituto di Applicazioni del Calcolo, Consiglio Nazionale delle Ricerche (CNR-IAC), Bari, Italy \\ ${ }^{2}$ Telespazio Spa, Centro di Geodesia Spaziale, Matera, Italy \\ ${ }^{3}$ Dipartimento di Ingegneria, Università di Ferrara, Ferrara, Italy \\ ${ }^{4}$ Agenzia Spaziale Italiana (ASI), Centro di Geodesia Spaziale, Matera, Italy
}

Received: 6 October 2004 - Revised: 4 January 2005 - Accepted: 3 March 2005 - Published: 18 March 2005

Part of Special Issue "Landslides and debris flows: analysis, monitoring, modeling and hazard"

\begin{abstract}
This work presents the first results of an experiment aiming to derive a high resolution Digital Terrain Model (DTM) by kinematic GPS surveying. The accuracy of the DTM depends on both the operational GPS precision and the density of GPS samples. The operational GPS precision, measured in the field, is about $10 \mathrm{~cm}$. A Monte Carlo analysis is performed to study the dependence of the DTM error on the sampling procedure. The outcome of this analysis is that the accuracy of the topographic reconstruction is less than $1 \mathrm{~m}$ even in areas with a density of samples as low as one sample per $100 \mathrm{~m}^{2}$, and becomes about $30 \mathrm{~cm}$ in areas with at least one sample per $10 \mathrm{~m}^{2}$. The kinematic GPS technique gives a means for a fast and accurate mapping of terrain surfaces with an extension of a few $\mathrm{km}^{2}$. Examples of application are the investigation of archaeological sites and the stability analysis of landslide prone areas.
\end{abstract}

\section{Introduction}

A representation of terrain morphology is essential to model geological processes occurring on the earth surface. Topographic data are currently available from land surveying, aerial photogrammetry (Mikhail et al., 2001; Heipke, 1995), optical (Toutin, 2001) and radiometer images (Hirano et al., 2003), synthetic aperture radar (SAR) (Farr and Kobrick, 2000; Rabus et al., 2003; Toutin and Gray, 2000), and light detecting and ranging (LIDAR) technique (Wehr and Lohr, 1999).

The USGS' National Elevation Dataset furnishes a seamless raster Digital Elevation Model (DEM) of USA territories with a spatial gridding ranging form 10 to $30 \mathrm{~m}$ and elevation units standardized to decimal meters (Gesch et al., 2002).

The processing of data acquired in the $\mathrm{C}$ band (radar wavelength $\lambda=5 \mathrm{~cm}$ ) by the SRTM interferometric radar on board of the Endeavor Shuttle provides a nominal $30 \mathrm{~m}$ DEM of

Correspondence to: P. Rutigliano

(paolo.rutigliano@asi.it) over $80 \%$ of the Earth landmass surface with an estimated vertical accuracy of $15 \mathrm{~m}$ and a discretization to the nearest meter. Outside USA the spatial resolution is reduced to $90 \mathrm{~m}$ pixels (Farr and Kobrick, 2000). A preliminary distribution of 1 and 3 arcsec topographic data (SRTM-1 and SRTM-3) has been made for evaluation purposes (Farr and Kobrick, 2000).

Stereo optical images acquired by IKONOS provides another means to obtain a DEM with planimetric and vertical accuracy of $1 \mathrm{~m}$. The successful processing of these images depends on the sun illumination and cloud covering conditions (Toutin and Cheng, 2000).

All the above space mapping techniques provide a DEM, which does not mean only topography but also tree and buildings height. However, often users need a high-resolution Digital Terrain Model (DTM) of a small area of interest. High resolution DTMs are needed, for example, in landslide prone areas to be used as updated topographic information in run-off models, and in the mapping of river beds for hydrological risk analysis. Recently, a ground-based radar interferometer has been proposed for the remote topographic mapping of small areas, able to produce a DTM with a spatial and vertical accuracy of a few meters (Nico et al., 2004; Nico et al., 2005). The application of GPS techniques to produce height profiles has been recently described in the literature (Baldi et al., 2000; Baldi et al., 2002).

In this paper, we study the use of kinematic GPS to generate the DTM of a whole topographic surface. The first results of an experiment aiming to produce high resolution, i.e. sub-metric, topographic maps of a small terrain surface are described. The measurement campaign has been carried out at the archaeological site of Torre di Satriano, close to the village of Satriano, Basilicata Region, Italy. This site is characterized by the presence of human settlements since the prehistoric age. An accurate and updated DTM of an archaeological site can provide useful information about the human settlement structures. The GPS survey technique provides an easy, quick, and comparatively cheap tool to extract accurate and high resolution topographic information. 


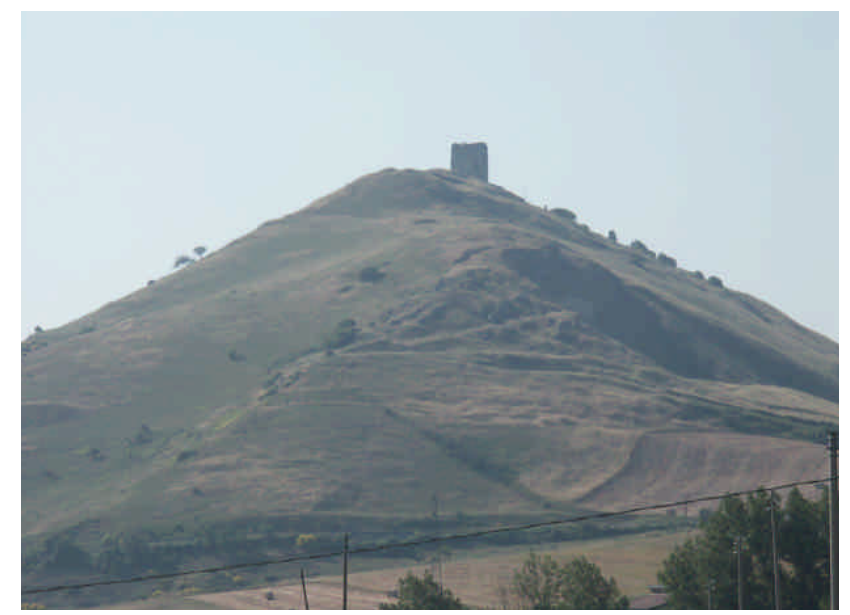

Fig. 1. View of the Torre di Satriano site. Ruines of the ancient Satrianum settlements are visible at the summit of this archaeological site. The picture shows the fairly irregular site morphology, characterized by a topographic height between 900 and $1000 \mathrm{~m}$ a.s.1., and an average slope of about $50 \%$.

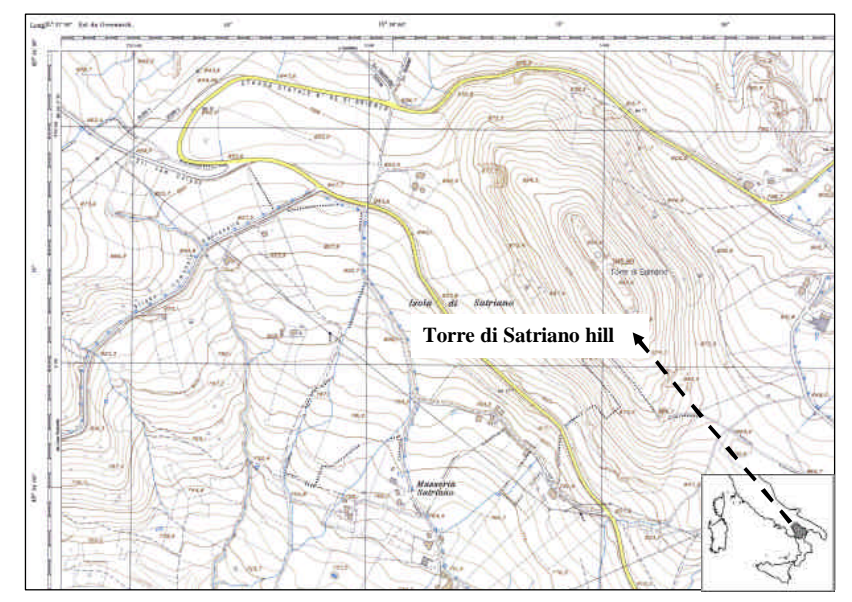

Fig. 2. Location of Torre di Satriano site.

A Triangulated Irregular Network (TIN) has been used to model GPS data. A TIN is able to represent the topographic information at a both small and large scale of resolution. Grid-based DTMs, even if easier to use, suffer the main disadvantage of representing the terrain surface at a constant resolution which in practice means the resolution of the smallest topographic feature of interest. Further, a gridbased representation makes difficult the modelling of geological processes with a significant horizontal component, such as stream meandering and landslides (Crosta et al., 2003).

The paper has the following structure. A description of the test-site area and of its archaeological relevance in given in Sect. 2. Section 3 is devoted to a short discussion about the GPS survey. Terrain interpolation methods based on TIN models are discussed in Sect. 4. In Sect. 5 the postprocessing of GPS data is detailed and results presented. Finally, some conclusions are drawn in Sect. 6.

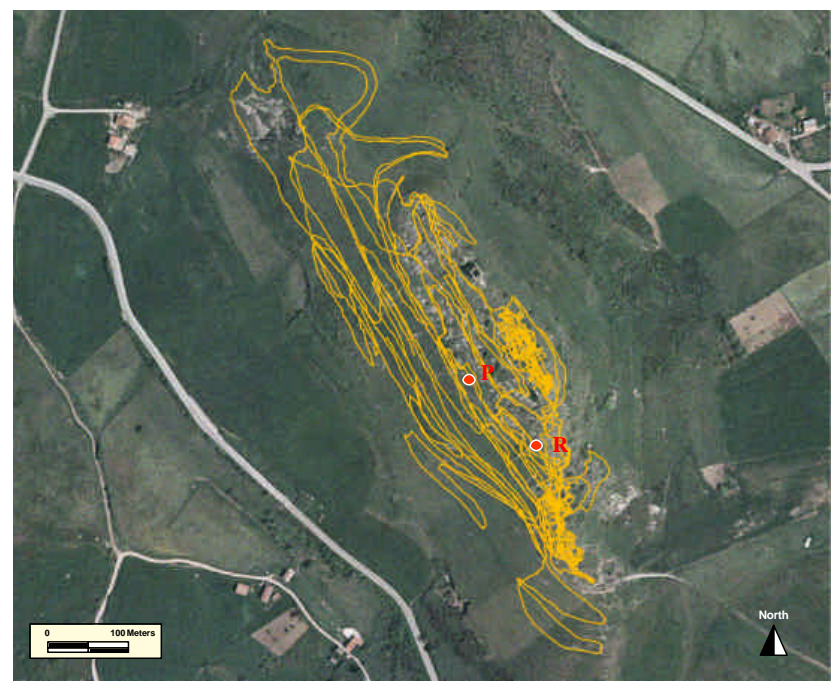

- Local reference stations used in the Kinematics surveys

Fig. 3. Location of GPS samples (yellow line) overlaid to a satellite optical image of the Torre di Satriano site. Red spots give the location of local reference stations used in the kinematic survey. The GPS reference station has been installed in point $P$. GPS rover receivers have been initialized in point $R$.

\section{Test-site}

The archaeological site of Torre di Satriano, close to the village of Satriano, Basilicata Region, Italy, has been chosen for the experiment. The Torre di Satriano site dominates a vast territory and still nowadays represents a clear landmark from a long distance (see Fig. 1). The site controls a system of crossroads which interconnects the area of MarmoPlatano with the geographical districts of the river-valleys of Ofanto, Agri and Vallo di Diano (see map in Fig. 2). The position as well as the presence of water springs and fertile lands, favoured the development of human settlements since the Bronze age. The imposing ruines still visible today on the summit of the Torre di Satriano hill, including the mighty tower, the rests of a cathedral and other small buildings on the slope side are related to the ancient Satrianum settlement, dating back to the Middle age. As shown in Fig. 1, the terrain morphology of the test site is fairly irregular, with a topographic height between 900 and $1000 \mathrm{~m}$ a.s.l., and an average slope of about $50 \%$.

\section{GPS survey}

The GPS campaign consisted of two steps: a static survey to establish a local GPS network, and a kinematic survey for the terrain mapping. All data have been collected using dualfrequency Trimble 4000 SSi GPS receivers, in five different daily campaigns from October 2003 to April 2004. 


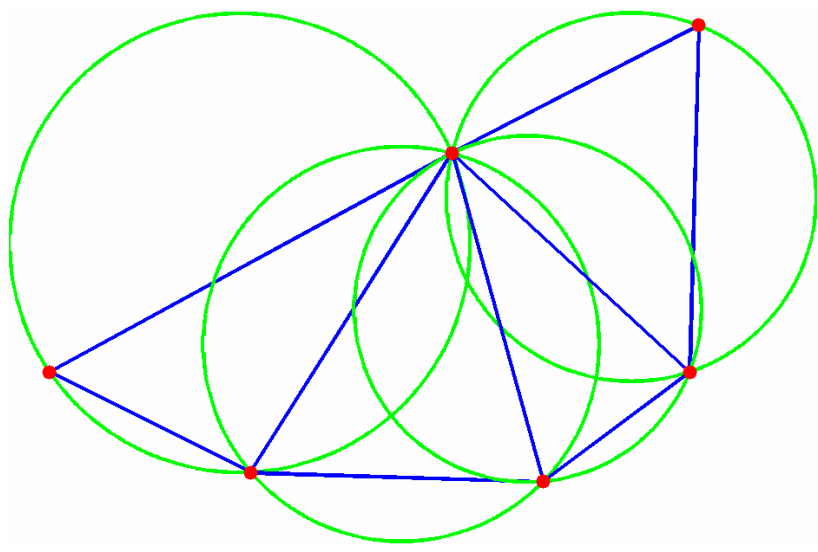

Fig. 4. Definition of Delaunay triangulation by means of the "empty circle criterion".

\subsection{Static survey}

The GPS local reference network has been established by means of four markers. Two of them, have been placed at the top of the hill, while the other two ones have been installed at the bottom of the hill. The position of each marker has been surveyed by static GPS in three different daily sessions, of about $8 \mathrm{~h}$ each, from October to November 2003. These data have been merged with those collected at the permanent GPS stations at Matera, Tito and Cagliari sites. These stations belong to the Italian GPS Reference Network, managed by the Italian Space Agency. The joint processing of data allows the Torre di Satriano local GPS network to be referenced within a largest network of permanent GPS stations. The two markers at the top of the hill, shown in Fig. 3, have been used during the kinematic survey sessions.

\subsection{Kinematic survey}

The kinematic survey has been performed in two different daily measurement campaigns in February and April 2004. The reference station has been installed in point $P$ (see Fig. 3). At the beginning of each kinematic session, GPS rover receivers have been initialized in point $R$ (see Fig. 3). During the survey, this point has been crossed 107 times along different paths. The cut-off angle of GPS antenna has been set to $15^{\circ}$. The GPS signal has been sampled at a rate of 2 samples per second. The minimum number of in-view satellites has been set to five. It is worth noting that obstacles, such as trees and walls, as well as the need to collect data along the flank of the hill, reduce the sky visibility of GPS antenna, so reducing the number of in-view GPS satellites and, as a consequence, the loss of GPS signal lock. In the two kinematic daily surveys, there has been a small number of interruptions as the cycle slip, i.e. the loss of GPS signal lock, has been very short. Each GPS rover acquired data for a time interval of about $3 \mathrm{~h}$, with an average number of 2 re-initializations.

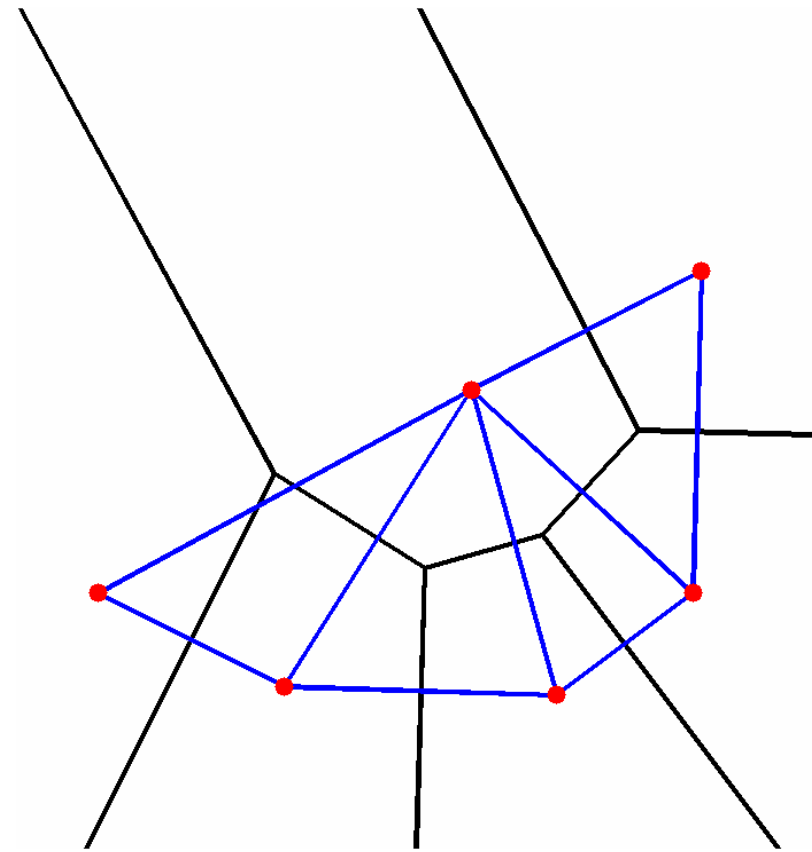

Fig. 5. Voronoi diagram and Delaunay triangulation corresponding to the set of nodes of Fig. 4

\section{Terrain representation}

A terrain surface can be represented in a $x y$ plane by a set of nodes which are the projection on the plane of terrain sampled points $P(x, y, z)$. A number of methods for triangulating a set of irregularly spaced nodes have been proposed (Kumler, 1994). They are at the basis of Triangular Irregular Network (TIN) models very popular among the Geographical Information Systems (GIS) currently available on the market. A TIN represents a topographic surface as a set of non-overlapping contiguous triangular facets of irregular size and shape (Burrough, 1986). Important information such as slope, aspect, contour and visibility maps, can be extracted from these models. Here, we focus on the commonly used Delaunay triangulation (DT) and its corresponding Voronoi tessellation (VT).

Given a set of nodes $N=\left\{\left(x_{i}, y_{i}\right) \in R^{2}\right\}_{1 \leq i \leq n}$ in the plane, a DT of $N$ is given by the empty circumcircle criterion (Tsai, 1993). This criterion, stating that the circumcircle passing trough the three vertices of a triangle does not contain any other sample point, is used to determine whether a triangle is Delaunay or not. Figure 4 depicts a set of six points and the corresponding DT emphasizing the empty circumcircle property of triangles. Every region, except for the external ones, is a triangle. The DT is unique and it maximizes the minimum internal angle of each triangle, so making them as equilateral as possible.

The DT of $N$ can also be defined in terms of the VT of the set $N$. The plane is uniquely subdivided into $\mathrm{n}$ convex polygonal regions called Voronoi (or Thiessen) polygons, each associated with a node. The Voronoi polygon $\pi_{i}$ of node $\left(x_{i}\right.$, 


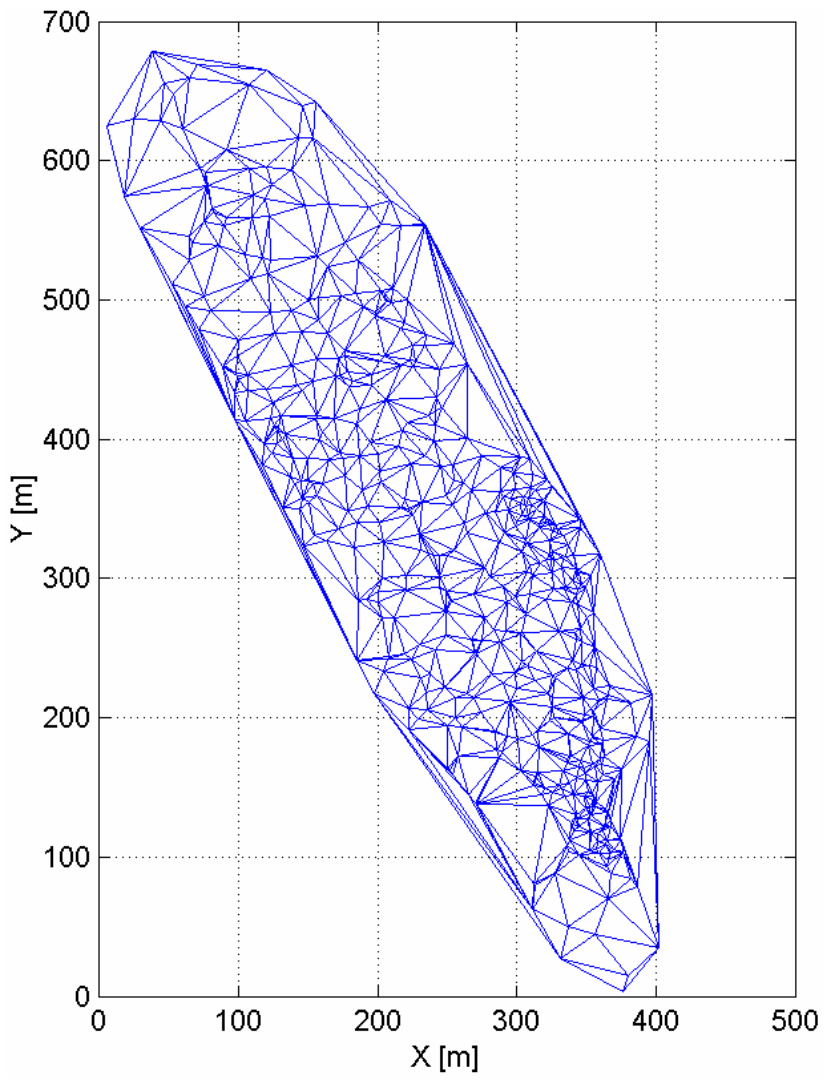

Fig. 6. Delaunay triangulation of GPS samples. A decimation factor equal to 100 has been applied to facilitate the interpretation of picture.

$\left.y_{i}\right)$ is the set of points $(x, y)$ in the plane lying closer to $\left(x_{i}\right.$, $y_{i}$ ) than to any other node of $N$, i.e.

$\pi_{i}=\left\{\left(x_{i}, y_{i}\right) \in R^{2} \mid \sqrt{\left(x-x_{i}\right)^{2}+\left(y-y_{i}\right)^{2}}<\right.$

$\left.\sqrt{\left(x-x_{j}\right)^{2}+\left(y-y_{j}\right)^{2}}\right\}_{1 \leq i \leq n} \forall \mathrm{g}=1, \ldots, n, i \neq j$.

The DT is the dual of the VT and is constructed by connecting the nodes whose associated Voronoi polygons share a common edge. The relationship between DT and VT is illustrated in Fig. 5. Many algorithms for constructing a DT or a VT have been developed (Guibas and Stolfi, 1985; Knuth et al, 1992; Lee and Schlachter, 1980). When the sample set is too dense, the triangulation is constructed by selecting a subset, the Very Important Points (VIP) that better represents the topographic surface (Lee, 1991).

Starting from a DT, an interpolation can be performed to fit the terrain surface. Each triangle can be described by a local polynomial surface whose parameters depend on vertices of the given triangle and of those close to it. A simple approach is the linear fitting consisting in computing the plane surface passing through the three vertices of triangle. This allows to derive the height $z$ of every point inside the triangle. If a smooth fit to the terrain surface is needed over every triangle,
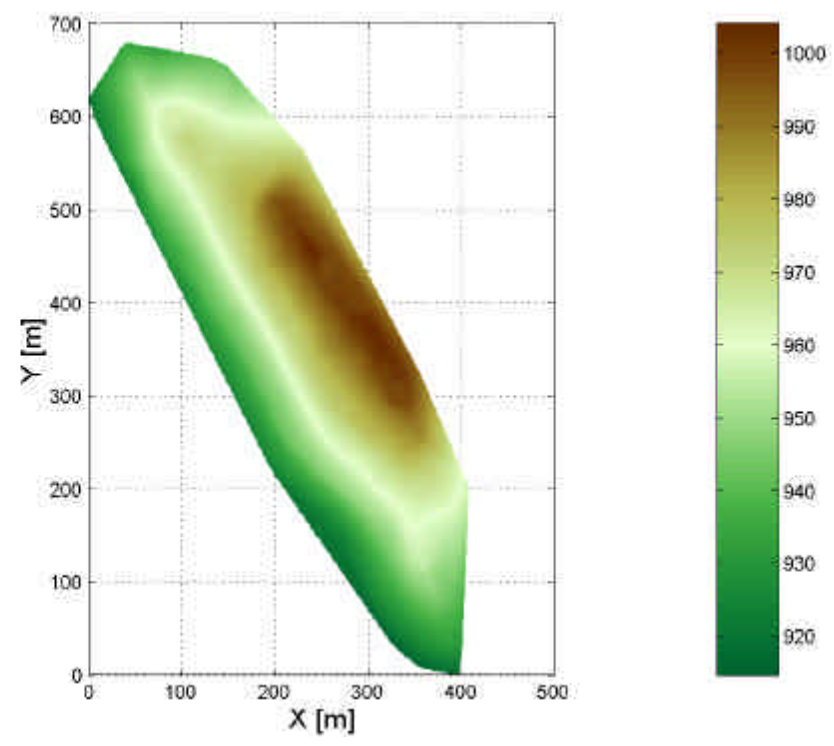

Fig. 7. Digital Terrain Model of the surveyed surface at the Torre di Satriano site. It has been derived by processing the GPS data collected in the measurement campaign.

polynomial functions are applied to both the triangle and its neighbours in order to get slopes and curvatures smoothly changing even across triangle edges (Akima, 1978; Lancaster and Salkauskas, 1986).

\section{Analysis of results}

A terrain surface of about $150 \cdot 10^{3} \mathrm{~m}^{2}$ has been surveyed during the measurement campaign. Figure 3 shows the extension of the surveyed area and the location of GPS data, overlaid to a satellite optical image of the Torre di Satriano hill. A number of 47000 samples has been collected in an overall time interval of $12 \mathrm{~h}$. The RMS of continuous kinematic GPS solution is below $1 \mathrm{~cm}$.

The operational precision of GPS measurements has been tested in the field by computing the dispersion of height values of the reference point $R$ measured at different times along the 107 different paths (see Sect. 3.2). The standard deviation of the height samples is about $8 \mathrm{~cm}$.

Figure 6 displays the location of GPS samples, decimated by a factor 100 to facilitate the interpretation of picture. Samples have been connected by a Delaunay triangulation. Terrain surface within each triangular facet has been reconstructed by a linear interpolation. The resulting DTM is displayed in Fig. 7.

Besides the operational GPS precision, the final accuracy of the topographic reconstruction depends on the sampling procedure. To study the impact of spatial distribution of samples on the final accuracy of the DTM, a Monte Carlo analysis has been performed. Synthetic GPS sample patterns have been generated within the studied area. For a given spatial density, namely the number of GPS samples per $\mathrm{m}^{2}$, the location of samples has been obtained by a uniform random 


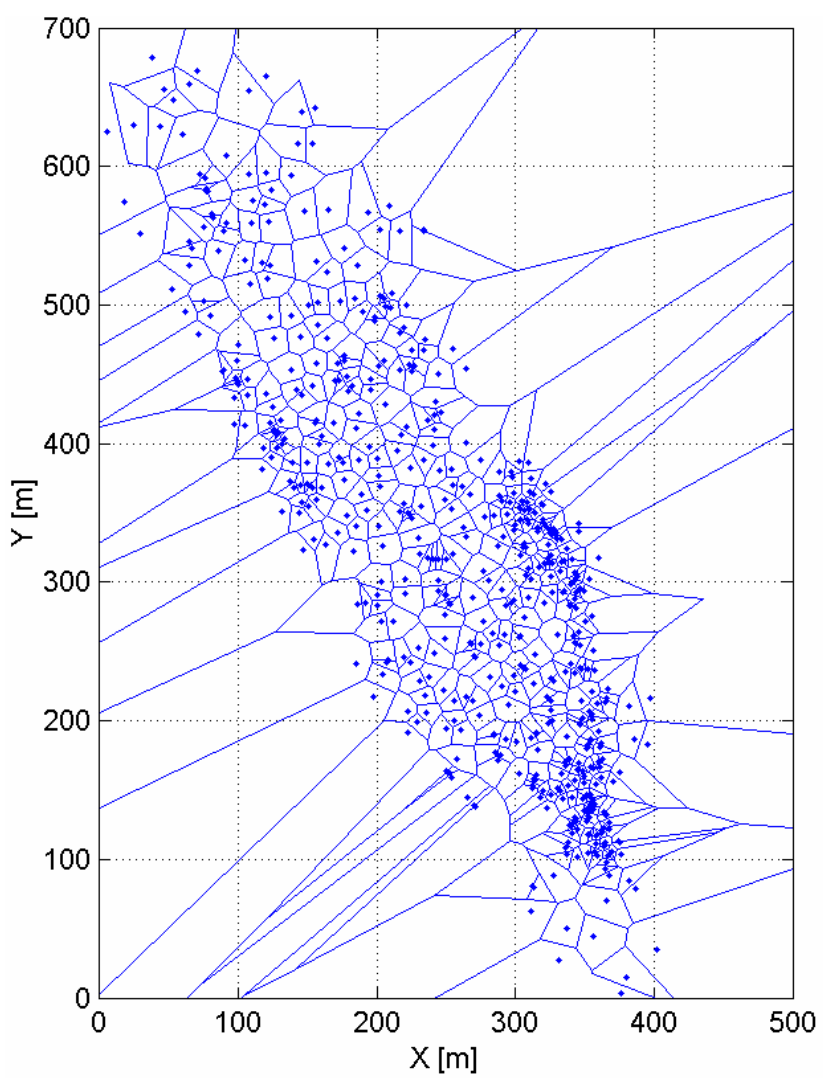

Fig. 8. Voronoi diagram of GPS samples. A decimation factor equal to 100 has been applied to facilitate the interpretation of picture.

distribution. The range of density values used in the Monte Carlo analysis has been estimated directly from the Voronoi tessellation of GPS samples. Each Voronoi polygon contains just one sample. Figure 8 depicts the Voronoi diagram corresponding to the experimental dataset, decimated by a factor 100. The majority of Voronoi polygons have an area smaller than $10 \mathrm{~m}^{2}$. This means the local density of samples, computed as the inverse of polygons' area value, is higher than $0.1 \mathrm{sample} / \mathrm{m}^{2}$. Voronoi polygons with the smallest area values are located in regions of high sample density. The frequency distribution of polygons' area values is plotted in Fig. 9. These values have been used to compute the density map of samples displayed in Fig. 10. Density values higher than $1 \mathrm{sample} / \mathrm{m}^{2}$ have been saturated to appreciate the lower density values into which we are interested in.

The dependence of the DTM accuracy on the sampling procedure has been studied in the density range $(0.001,0.1)$ samples $/ \mathrm{m}^{2}$. For a given density value, patterns of synthetic GPS samples have been generated, having a random location and subject to the constraint of a constant average density. The height value corresponding to each sample has been computed by interpolation from the reconstructed DTM of Fig. 7. A gaussian random noise, with standard deviation of $10 \mathrm{~cm}$ has been added to the interpolated height values to accounting for the operational GPS precision. This synthetic GPS dataset has been then used to reconstruct a new topo-

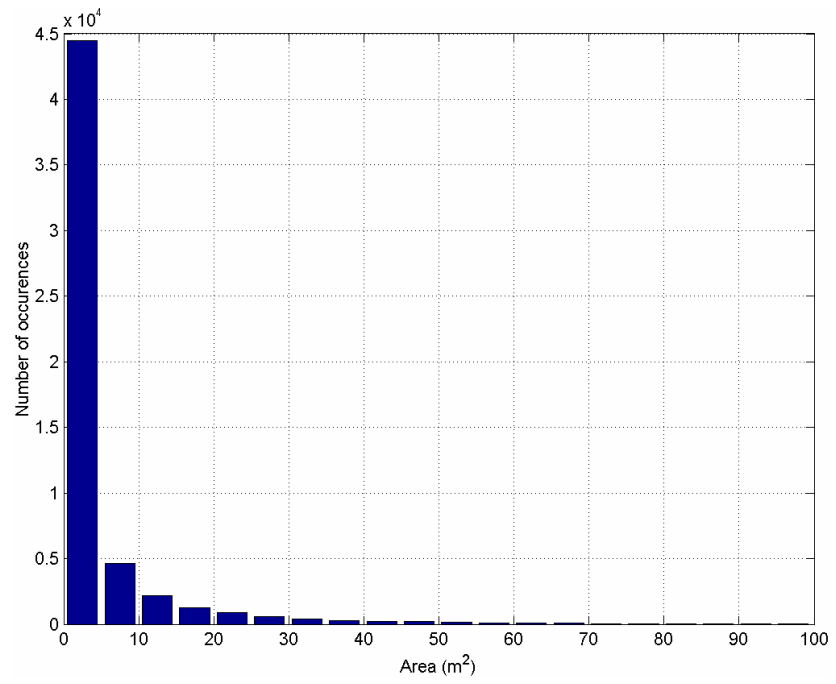

Fig. 9. Frequency distribution of the area values of Voronoi polygons.

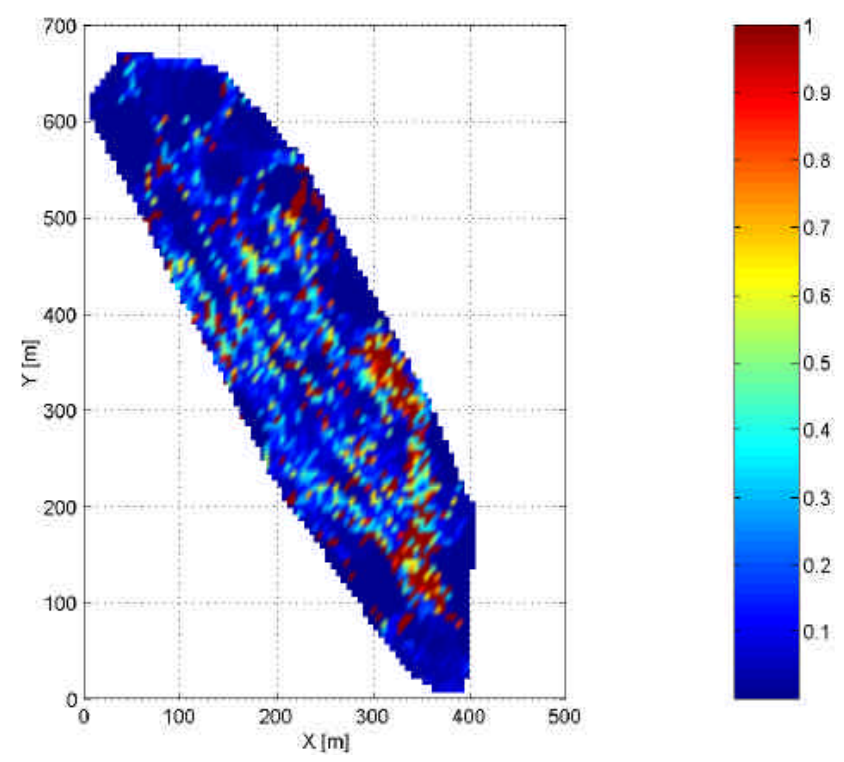

Fig. 10. Density map (sample/m²) of GPS samples.

graphic surface following the procedure explained at the beginning of this section. Differences between the two surfaces depends on the joint effect of operational GPS precision, i.e. the noise level, and sample density. The mean and standard deviation of absolute height differences, averaged over different realizations of the synthetic pattern of GPS samples, have been computed for each density value. Figure 11 shows the mean value and standard deviation for sample densities lower than $0.1 \mathrm{samples} / \mathrm{m}^{2}$. In particular, it has been found that the accuracy of the topographic reconstruction is about $0.5 \mathrm{~m}$ and it is less than $1 \mathrm{~m}$ even in areas with a sample density lower than $0.01 \mathrm{samples} / \mathrm{m}^{2}$. This figure has been estimated using the $2 \sigma$ standard deviation around the mean of absolute height differences. 


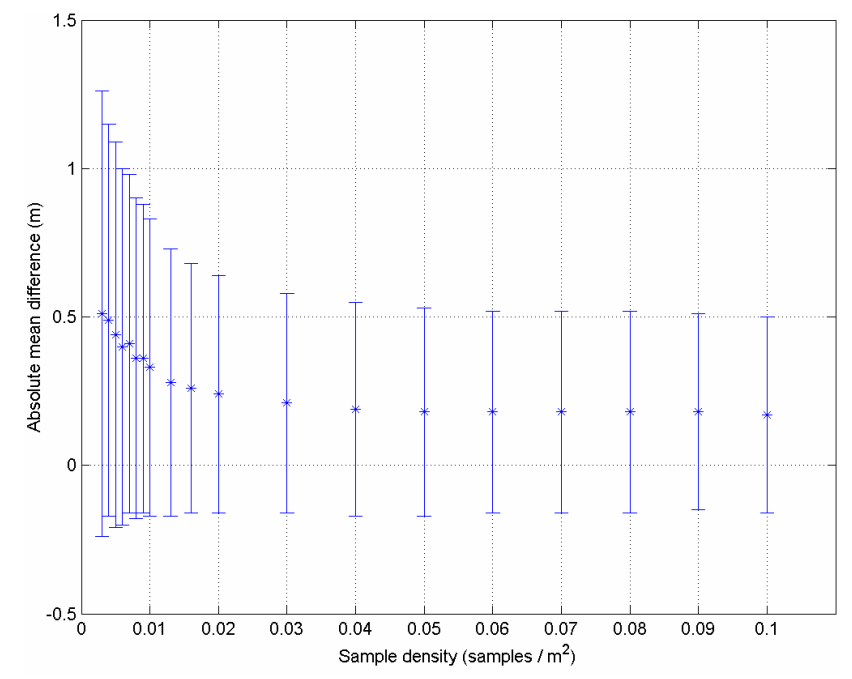

Fig. 11. Absolute mean difference of topographic reconstruction vs. density of GPS samples.

\section{Conclusions}

A procedure to derive a high resolution DTM of small terrain surfaces by means of kinematic GPS data has been described. The accuracy of the DTM depends on the operational GPS precision and the density of GPS samples. The operational GPS precision, measured in the field, is in the order of a few centimetres. The sparse GPS samples have been connected with a Delaunay triangulation and terrain surface within each triangle reconstructed by interpolation. A Monte Carlo analysis has been performed to study the dependence of the DTM error on the spatial distribution of GPS samples. The outcome of this analysis is that the topographic reconstruction accuracy is less than $1 \mathrm{~m}$ even in areas with a density of samples as low as one sample per $100 \mathrm{~m}^{2}$.

The main advantage of the proposed technique is its capability of producing high-resolution DTMs of small terrain surfaces with a fast and comparatively cheap measurement campaign. An application to the terrain mapping of archaeological sites has been presented. Other examples of application of the GPS technique are the derivation of updated high resolution DTMs in landslide prone areas, needed as an input in run-off models, and the mapping of river beds for hydrological risk analysis.

Acknowledgements. The authors are grateful to M. Osanna and M. Di Lieto (School of Specialization in Archaeology, University of Basilicata, Matera, Italy) for valuable discussions and support to the experimental campaign.

Edited by: M. Jaboyedoff

Reviewed by: C. Gerstenecker and another referee

\section{References}

Akima, H.: A method of bivariate interpolation and smooth surface fitting for values given at irregularly distributed points, ACM Transactions on Mathematical Software, 4, 148-159, 1978.

Baldi, P., Bonvalot, S., Briole, P., and Marsella, M.: Digital photogrammetry and kinematic GPS applied to the monitoring of Vulcano Island, Aeolian Arc, Italy, Geophys. J. Int., 142, 801811, 2000.

Baldi, P., Bonvalot, S., Briole, P., Coltelli, M., Gwinner, K., Marsella, M., Puglisi, G., and Remy, D.: Validation and comparison of different techniques for the derivation of digital elevation models and volcanic monitoring (Vulcano Island, Italy), Int. J. Rem. Sens., 23, 22, 4783-4800, 2002.

Burrough, P. A.: Principles of Geographical Information Systems for Land Resources Assessment, Clarendon Press, Oxford, 1986.

Crosta, G.B., Imposimato, S., and Roddeman, D.G.: Numerical modelling of large landslides stability and runout, Natural Hazards and Earth System Sciences, 3, 523-538, 2003,

SRef-ID: 1684-9981/nhess/2003-3-523.

Farr, T. G. and Kobrick, M.: Shuttle radar topography mission produces a wealth of data, Eos Transactions, AGU, 81, 48, 583-585, 2000.

Gesch, D., Oimoen, M., Greenlee, S., Nelson, C., Steuck, M., and Tyler, D.: The national elevation dataset, Photogrammetric Engineering and Remote Sensing, 68, 1, 5-15, 2002.

Guibas, L. and Stolfi, J.: Primitives for the manipulation of general subdivisions and the computation of Voronoi diagrams, ACM Trans. Graphics, 4, 72-123, 1985.

Heipke, C.: State-of-the-art of Digital Photogrammetric Workstations for topographic applications, Photogrammetric Engineering and Remote Sensing, 61, 1, 49-56, 1995.

Hirano, A., Welch, R., and Lang, H.: Mapping from ASTER stereo image data: DEM validation and accuracy assessment, ISPRS Journal of Photogrammetry and Remote Sensing, 57, 5-6, 356370, 2003.

Lancaster, P. and Salkauskas, K.: Curve and Surface fitting: An introduction, London Academic Press, London, 1986.

Lee, D. T. and Schlachter, B. J.: Two algorithms for constructing a Delaunay triangulation, Int. J. Comp. Informat. Sci., 9, 3, 219242, 1980.

Lee, J.: Comparison of existing methods for building triangular irregular network models of terrain from grid digital elevation nodes, Int. J. Geogr. Inform. Sys., 5, 267-285, 1991.

Knuth, D., Guibas, L., and Shamir, M.: Randomized incremental construction of Delaunay and Voronoi diagrams, Algorithmica, 7, 4, 381-413, 1992.

Kumler M. P.: An intensive comparison of triangulated irregular networks (TINs) and digital elevation models (DEMs), Cartographica, 31, 2, Monograph 45, 1-48, 1994.

Mikhail, E., McGlone, C., and Bethel, J. S.: Introduction to modern photogrammetry, Wiley John \& Sons Inc., 2001.

Nico, G., Leva, D., Antonello, G., and Tarchi D.: Ground-based SAR interferometry for terrain mapping: theory and sensitivity analysis, IEEE Transactions on Geoscience and Remote Sensing, 42, 6, 1344-1350, 2004.

Nico, G., Leva, D., Fortuny, J., Antonello, G., and Tarchi, D.: Generating Digital Terrain Models by a ground-based Synthetic Aperture Radar interferometer, IEEE Transactions on Geoscience and Remote Sensing, 43, 1, 45-49, 2005. 
Rabus, B., Eineder, M., Roth, A., and Bamler, R.: The shuttle radar topography mission - a new class of digital elevation models acquired by spaceborne radar, ISPRS Journal of Photogrammetry and Remote Sensing, 57, 4, 241-262, 2003.

Tsai, V. J. D.: Delaunay triangulation in TIN creation: an overview and linear-term algorithm, International Journal of Geographical Information Systems, 7, 501-524, 1993.

Toutin, T.: Elevation modelling from satellite visible and infrared (VIR) data, Int. J. Rem. Sens., 22, 6, 1097-1125, 2001.
Toutin, T. and Gray, L.: State-of-the-art of elevation extraction from satellite SAR data, J. Photogram. Rem. Sens., 55, 1, 13-33, 2000.

Toutin, T. and Cheng, P.: DEM with stereo IKONOS: a reality if ..., Earth Observation Magazine, 9, 7, 17-21, 2000.

Wehr, A. and Lohr, U.: Airborne laser scanning - an introduction and overview, ISPRS Journal of Photogrammetry and Remote Sensing, 54, 2-3, 68-82, 1999. 